\title{
An X-ray view of Mrk 705 (Research Note)
}

\section{A borderline narrow-line Seyfert 1 galaxy}

\author{
L. C. Gallo ${ }^{1}$, I. Balestra ${ }^{1}$, E. Costantini ${ }^{2,3}$, Th. Boller ${ }^{1}$, V. Burwitz ${ }^{1}$, E. Ferrero ${ }^{4}$, and S. Mathur ${ }^{5}$
}

\author{
1 Max-Planck-Institut für extraterrestrische Physik, Postfach 1312, 85741 Garching, Germany \\ e-mail: lgallo@mpe.mpg.de \\ 2 SRON National Institute for Space Research Sorbonnelaan 2, 3584 CA Utrecht, The Netherlands \\ 3 Astronomical Institute, Utrecht University, PO Box 80000, 3508 TA, Utrecht, The Netherlands \\ ${ }^{4}$ Landessternwarte Heidelberg, Königstuhl 12, 69117, Heidelberg, Germany \\ 5 Department of Astronomy, The Ohio State University, 140 West 18th Avenue, Columbus, OH 43210, USA
}

Received 22 July 2005 / Accepted 3 August 2005

\section{ABSTRACT}

Mrk 705 exhibits optical properties of both narrow- and broad-line Seyfert 1 galaxies. We examine the X-ray properties of this borderline object utilising proprietary and public data from Chandra, ASCA, ROSAT and RXTE, spanning more than twelve years. Though long-term flux variability from the pointed observations appears rather modest $(\sim 3 \times)$, we do find examples of rare large amplitude outbursts in the $R X T E$ monitoring data. There is very little evidence of long-term spectral variability as the low- and high-energy spectra appear constant with time. A $\sim 6.4 \mathrm{keV}$ emission line is detected in the ASCA spectra of Mrk 705, but not during the later, higher flux state Chandra observation. However, the upper limit on the equivalent width of a line in the Chandra spectrum is consistent with a constant-flux emission line and a brighter continuum, suggesting that the line is emitted from distant material such as the putative torus. Overall, the X-ray properties of Mrk 705 appear typical of BLS1 activity.

Key words. galaxies: active - galaxies: individual: Mrk 705 - galaxies: nuclei - X-rays: galaxies

\section{Introduction}

Narrow-line Seyfert 1 (NLS1) galaxies are defined on the bases of their optical properties, primarily that they possess narrow permitted lines, weak [O III] and strong Fe II (Osterbrock \& Pogge 1985; Goodrich 1989). It was realised that these objects often exhibit extreme X-ray behaviour as well (e.g. Puchnarewicz et al. 1992; Boller et al. 1996), such as steep spectra and rapid, large amplitude variability.

However, the traditional definition has become blurred by the discovery of objects which are not NLS1, but exhibit characteristic X-ray behaviour; and objects which are NLS1, but do not behave as such (e.g. Reeves et al. 2002; Williams et al. 2002, 2004; Dewangan \& Griffiths 2005). It has been suggested that NLS1 activity is an evolutionary phase that all active galaxies go through (e.g. Grupe 1996; Mathur 2000), and there is growing evidence in support of this conjecture. NLS1 and broad-line Seyfert 1s (BLS1) appear to follow different loci on the black hole mass - bulge velocity dispersion plane, implying that black holes in NLS1 are still growing (Mathur et al. 2001; Grupe \& Mathur 2004; Mathur \& Grupe 2005a,b). As such, it is important to examine objects which could be in a transition phase between NLS1 and BLS1.

In consideration of the optical spectrum, Mrk 705 is usually defined as an NLS1. By virtue of also being a relatively nearby $(z=0.029)$ PG quasar (PG 0923+129), and X-ray bright in the ROSAT All-Sky Survey (RASS; Voges et al. 1999), it is often found in many sample studies of AGN behaviour, but until now it has not benefited from a dedicated examination.

Mrk 705 would perhaps be better classified as an intermediate-type NLS1. The optical properties appear to be on the border that distinguishes NLS1 from BLS1. For example, the $F W H M$ of the $\mathrm{H} \beta$ emission line is approximately $1770-2150 \mathrm{~km} \mathrm{~s}^{-1}$ (e.g. Boroson \& Green 1992; Véron-Cetty et al. 2001; Botte 2004), and the [O III] emission is relatively strong $\left(\frac{[\mathrm{OIII}]}{\mathrm{H} \beta} \approx 2.4\right)$. Between $2-10 \mathrm{keV}$ the spectrum is somewhat steeper (see Sect. 4) than the canonical $\Gamma \approx 1.9$ found in BLS1 (e.g. Porquet et al. 2004) On the other hand, the low-energy X-ray spectrum, as observed 
Table 1. X-ray spectroscopy of Mrk 705. The telescope and instruments used are given in Cols. (1) and (2), respectively. The start date of the observation is shown in Col. (3). Usable energy range, exposure and total counts (source and background) are given in the following columns.

\begin{tabular}{lccccc}
\hline \hline $\begin{array}{l}\text { Observatory } \\
\text { Onst. }\end{array}$ & $\begin{array}{c}(3) \\
\text { Date } \\
\text { year.mm.dd }\end{array}$ & $\begin{array}{c}(4) \\
\text { Energy } \\
(\mathrm{keV})\end{array}$ & $\begin{array}{c}(5) \\
\text { Exp. } \\
(\mathrm{s})\end{array}$ & $\begin{array}{c}(6) \\
\text { Counts } \\
(\mathrm{cts})\end{array}$ \\
\hline ROSAT & PSPC & 1992.11 .05 & $0.1-2.4$ & 3855 & 5510 \\
\hline RSCAT & PSPC & 1993.26 .04 & $0.1-2.4$ & 5245 & 5652 \\
& SIS0 & 1998.14 .11 & $0.7-10$ & 23904 & 13746 \\
& SIS1 & & $0.7-10$ & 20844 & 9536 \\
& GIS2 & & $1-10$ & 29068 & 7871 \\
& GIS3 & & $1-10$ & 29068 & 9242 \\
\hline Chandra & 0-order & 2004.18 .03 & $0.5-7.5$ & 20893 & 3545 \\
ASIS-S & MEG & & $0.6-5$ & 20991 & 5989 \\
& HEG & & $0.8-7.5$ & 20991 & 2559 \\
\hline
\end{tabular}

with ROSAT, is relatively flat and hard ${ }^{1}$ compared to NLS1 (e.g. Wang et al. 1996; Grupe 1996; this study), but typical for BLS1. Mrk 705 was the only NLS1 that exhibited a significant hard spectrum at the time Grupe 1996 selected the soft X-ray AGN sample.

In comparing the known properties of Mrk 705 with a sample of NLS1 and BLS1 (Grupe \& Mathur 2004) we find several discrepancies. Mrk 705 has a flat $0.1-2.4 \mathrm{keV}$ spectral index more typical of BLS1. Like NLS1, it does possess strong $\mathrm{Fe}$ II emission (in terms of equivalent width), but with respect to $\mathrm{H} \beta$ the relative $\mathrm{Fe}$ II emission is actually weak (comparable to BLS1). Finally, as with the $\mathrm{H} \beta$ emission line width, $L / L_{\text {Edd }}$ (Baskin \& Laor 2004) lies in the region occupied by both NLS1 and BLS1.

We report the first dedicated Chandra X-ray observation of Mrk 705, obtained through the Guaranteed Time programme. In addition we make use of archival ROSAT, ASCA and RXTE data to examine the X-ray (0.1-10 keV) properties of Mrk 705 in detail.

\section{Observations and data reduction}

X-ray spectroscopic observations of Mrk 705, which are either proprietary to us or publicly available, have been performed with ROSAT, Chandra and ASCA. The spectroscopic data utilised in this analysis are described in Table 1.

In addition, Mrk 705 is observed nearly daily with the RXTE All-Sky Monitor (ASM). Quick-look results provided by the ASM/RXTE team are briefly discussed in Sect. 5.

1 The hardness ratio $(H R)$ used in this paper will be defined as $H-S / H+S$, where $H$ and $S$ are count rates in a hard and soft energy band, respectively. During the ROSAT era $H=0.4-2.4 \mathrm{keV}$ and $S=0.1-0.4 \mathrm{keV}$ were typically used. We will adopt this convention unless stated otherwise (as in Sect. 5.2).

\subsection{Chandra observation}

Mrk 705 was observed with Chandra on 2004 March 18 with the Advanced CCD Imaging Spectrometer (ACIS-S) for about $21 \mathrm{ks}$. To minimise pile-up effects on the ACIS image, a grating was inserted in the photon path; the High-Energy Transmission Grating Spectrometer (HETGS) was used to disperse photons and so to reduce the count rate on the zeroth order image Unfortunately, pile-up was still problematic at about the 30\% level. Accordingly, a pile-up model (Davis 2001) was adopted in all spectral fits.

Data were processed with the Chandra Interactive Analysis of Observations software (CIAO 3.2), using the Chandra Calibration Database (CALDB 3.0.0). We applied the recently released, time-dependent gain correction ${ }^{2}$, which is necessary to adjust the "effective gains", which drift with time due to an increasing charge transfer inefficiency (CTI).

The data were filtered to include only the standard event grades $0,2,3,4$ and 6 . We filtered time intervals with high background by performing a $3 \sigma$ clipping of the background level using the script analyze_ltcrv ${ }^{3}$. This yielded an effective exposure time of about $21 \mathrm{ks}$ for the ACIS-S3 chip in the energy range $0.3-10 \mathrm{keV}$.

The background was selected from an off-source circular region of radius $50^{\prime \prime}$. To extract the zeroth and higher order grating spectra we followed the standard procedures. We analyse the 0 -order spectrum as well as the combined (negative and positive) 1st-order spectra from the Medium Energy Grating (MEG) and the High Energy Grating (HEG).

\subsection{ASCA observation}

ASCA observed Mrk 705 for a total of 83 ks during which time all instruments were functioning normally. The processed data from all the ASCA instruments (SIS0, SIS1, GIS2, GIS3) were obtained from the TARTARUS website ${ }^{4}$ (version 3.0), where all the information describing the processing procedures can be found. The total exposure utilised for this observation is between 20-30 ks depending on the instrument (see Table 1).

\subsection{ROSAT observations}

Mrk 705 was observed with ROSAT on numerous occasions as part of the RASS or because it fell in the fieldof-view of other pointings. Two dedicated observations of Mrk 705 with the Position Sensitive Proportional Counter (PSPC; Pfeffermann et al. 1987) on ROSAT were performed about one year apart with the AGN viewed on-axis. Details of the observations are found in Table 1 .

The data were processed in the standard manner with EXSAS v030CT (Zimmermann et al. 1994). Spectra were created from source plus background counts extracted from a circular region with a radius of 2 arcmin and centred on the source. Background counts were extracted from a larger

\footnotetext{
${ }^{2}$ http://asc.harvard.edu/ciao/threads/acistimegain/

3 http://cxc.harvard.edu /ciao/threads/filter_ltcrv/

${ }^{4}$ http://astro.ic.ac.uk/Research/Tartarus/
} 


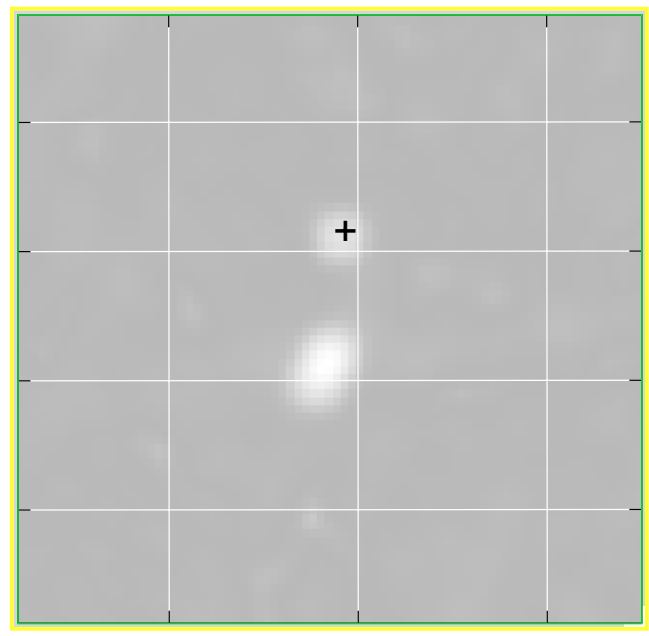

Fig. 1. The cross marks the Chandra X-ray position of Mrk 705 overplotted on the FIRST image. Right ascension $(\alpha)$ increases in the negative direction along the abscissa. Declination $(\delta)$ increases upward along the ordinate. The grid is divided into $30^{\prime \prime}$ in declination and $3 \mathrm{~s}$ in right ascension. The elongated radio source located about $30^{\prime \prime}$ from Mrk 705 is not detected with Chandra in the X-rays.

off-source region and appropriately scaled to the source cell size. Spectral files were then converted into FITS format so that the data could be analysed in XSPEC.

\section{X-ray coordinates}

The high-resolution capabilities of Chandra allows us to determine the X-ray position of Mrk 705 with arcsecond precision. The X-rays originate from a point source with coordinates $\alpha=09^{\mathrm{h}} 26^{\mathrm{m}} 03^{\mathrm{s}} .3$ and $\delta=+12^{\circ} 44^{\prime} 04^{\prime \prime} .2$, which agree well with the optical position of the AGN (Clements 1981). In comparing the Chandra image with the PSF expected from the observation, we found no extended X-ray emission within the spatial resolution of the instrument $(>800 \mathrm{pc}$ at the redshift of the source).

In Fig. 1 the Chandra coordinates are over-plotted on the archival $20 \mathrm{~cm}$ FIRST $^{5}$ (White et al. 1997) image obtained with the VLA. Although Mrk 705 is considered radio-quiet it is detected in the sensitive FIRST survey with an integrated flux density of about $8.5 \mathrm{mJy}$. The X-ray position is also in good agreement with the origin of the radio emission.

Of interest may be the elongated radio source located $\sim 30^{\prime \prime}$ south of Mrk 705. In the low-resolution instruments (i.e. ROSAT and ASCA), where the source extraction region is typically arcminutes in size, this source could contribute to the X-ray flux of Mrk 705. Little is known about this object other than that it is associated with the infrared source 2MASX J09260351+1243341, with a $K$-band flux density of $2.52 \pm 0.40 \mathrm{mJy}$, and a FIRST $(1.4 \mathrm{GHz})$ integrated flux density of $27.5 \pm 0.37 \mathrm{mJy}$.

At the redshift of Mrk 705, 2MASX J09260351+1243341 would be located $\approx 24 \mathrm{kpc}$ from the AGN. We considered the possibility that this could be a radio jet associated

\footnotetext{
5 http://sundog.stsci.edu/
}

with Mrk 705. However, the infrared emission, assuming a jet with a power-law spectrum $\left(F_{v} \propto v^{-\alpha}\right)$ and typical spectral index in the range 0.5-0.8 (e.g. Ferrari 1998; Worrall \& Birkinshaw 2004), is at least 25 times too high. More importantly, the object is not detected in the $\sim 20 \mathrm{ks}$ Chandra exposure, indicating that the X-ray fluxes measured with ASCA and ROSAT are dominated by Mrk 705.

\section{Spectral analysis}

The energy band in which the data from each instrument is analysed is given in Table 1 . All of the spectra were grouped such that each bin contained at least 20 counts. Spectral fitting was performed with XSPEC v11.3.1 (Arnaud 1996) and fit parameters are reported in the rest frame of the object. The quoted errors on the model parameters correspond to a $90 \%$ confidence level for one interesting parameter (i.e. a $\Delta \chi^{2}=2.7$ criterion). K-corrected luminosities were derived assuming isotropic emission, a value for the Hubble constant of $H_{0}=70 \mathrm{~km} \mathrm{~s}^{-1} \mathrm{Mpc}^{-1}$, and a standard cosmology with $\Omega_{\mathrm{M}}=0.3$ and $\Omega_{\Lambda}=0.7$. The value of the Galactic column density toward Mrk 705 was taken to be $N_{\mathrm{H}}=4.03 \times 10^{20} \mathrm{~cm}^{-2}$ (Elvis et al. 1989).

\subsection{The ROSAT spectra}

The PSPC is sensitive in the $0.1-2.4 \mathrm{keV}$ range and this offers the opportunity to examine lower levels of absorption than possible with $A S C A$ or Chandra. First attempts were made to fit the ROSAT spectra of Mrk 705 with a power-law modified by a neutral hydrogen column. Leaving the absorption component free to vary resulted in measurements consistent with the value given by Elvis et al. (1989). Fixing the photoelectric absorption to the Galactic value and including an additional component to estimate cold absorption intrinsic to the source did not enhance the fits or result in any meaningful detection of intrinsic absorption. As such, in subsequent fits (in particular with the ASCA and Chandra data) the photoelectric absorption component was simply fixed to the Galactic value.

A simple power-law was a reasonable fit to the 1993 (second) observation of Mrk 705 (see Fig. 2 and Table 2). Including additional components to mimic a multi-component continuum did not improve the simple model. However, the 1992 (first) PSPC observation appeared more complicated. A simple power-law provided a mediocre fit $\left(\chi_{v}^{2} /\right.$ d.o.f. $\left.=1.50 / 38\right)$ to the 0.1-2.4 keVdata. Multi-component models (e.g. broken power-law, and blackbody plus power-law) provided a substantial improvement over the single power-law (Table 2).

The most apparent difference between the two ROSAT observations is that the 1992 spectrum requires a second component in addition to a power-law. However, the hardness ratio at both epochs is comparable $\left(H R_{1992}=0.29 \pm 0.07\right.$, $\left.H R_{1993}=0.29 \pm 0.03\right)$, indicating that the spectral differences between the two observations may not be as significant as suggested by the modelling. Indeed, the primary differences in the two spectra are between $2-2.4 \mathrm{keV}$, where the sensitivity of the instrument begins to diminish. Therefore, it is not 


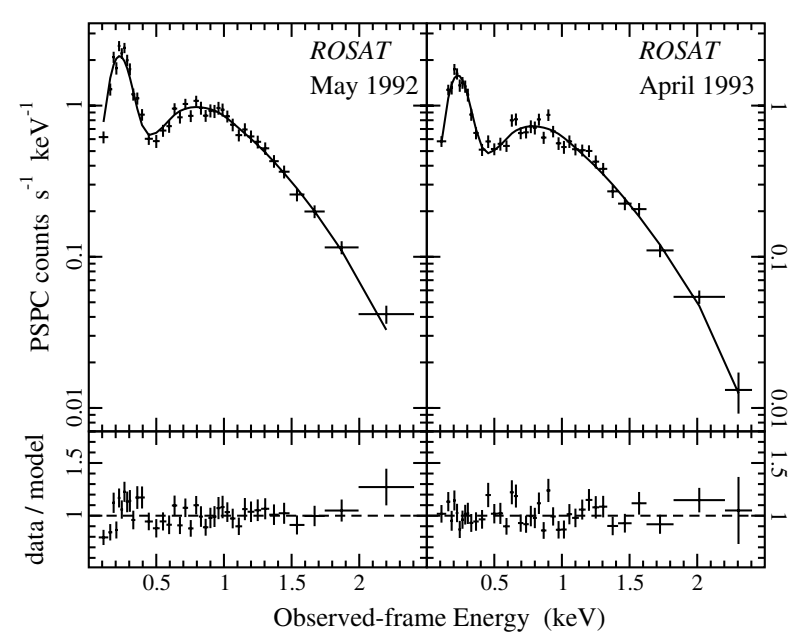

Fig. 2. The PSPC count rate spectrum fitted with a power-law with Galactic absorption (upper panels). The fit ratios are shown in the lower panels. The spectrum from the 1992 observation is shown on the left and from the 1993 observation on the right.

Table 2. ROSAT PSPC (0.1-2.4 keV) spectral fit results. The model and fit parameters are given in Cols. (1) and (2), respectively. The values of the parameters during the first (1992) and second (1993) observation are given in Cols. (3) and (4), respectively. Unabsorbed flux $(F)$ and luminosity $(L)$ are given for the power-law fit in units of $10^{-11} \mathrm{erg} \mathrm{cm}^{-2} \mathrm{~s}^{-1}$ and $10^{43} \mathrm{erg} \mathrm{s}^{-1}$, respectively. The values marked with $u$ indicate that the parameter is unconstrained and accurate uncertainties could not be estimated.

\begin{tabular}{lccc}
\hline \hline Model & $(2)$ & $(3)$ & $(4)$ \\
& Parameters & $\begin{array}{c}\text { Obs 1 } \\
(1992)\end{array}$ & $\begin{array}{c}\text { Obs 2 } \\
(1993)\end{array}$ \\
\hline power-law & $\chi_{v}^{2} /$ d.o.f. & $1.50 / 38$ & $1.20 / 38$ \\
& $\Gamma$ & $2.49 \pm 0.04$ & $2.51 \pm 0.04$ \\
& $F$ & 3.81 & 2.85 \\
& $L$ & 7.45 & 5.58 \\
\hline broken & $\chi_{v}^{2} /$ d.o.f. & $1.41 / 36$ & $1.24 / 36$ \\
power-law & $\Gamma_{1}$ & $2.61 \pm 0.06$ & $2.52 \pm 0.03$ \\
& $E_{\mathrm{b}}(\mathrm{keV})$ & $0.61 \pm 0.13$ & $1.70^{u}$ \\
& $\Gamma_{2}$ & $2.33 \pm 0.08$ & $1.50^{u}$ \\
\hline blackbody & $\chi_{v}^{2} /$ d.o.f. & $1.34 / 36$ & $1.25 / 36$ \\
plus & $k T(\mathrm{eV})$ & $62_{-20}^{+13}$ & $80_{-36}^{+25}$ \\
power-law & $\Gamma$ & $2.29_{-0.20}^{+0.06}$ & $2.45 \pm 0.04$ \\
\hline
\end{tabular}

conclusive whether apparent changes are due to intrinsic variability in the AGN or signal-to-noise.

Results from modelling the ROSAT PSPC data of Mrk 705 are tabulated in Table 2.

\subsection{The ASCA spectra}

Modelling the data from the four ASCA instruments separately revealed relatively good agreement in the measured fit parameters (within 90 per cent confidence levels). As such, the data from all the instruments were fitted simultaneously while allowing for an energy-independent scale factor between the SIS and GIS spectra. The residuals from each instrument were examined separately to ensure agreement. Since there was no

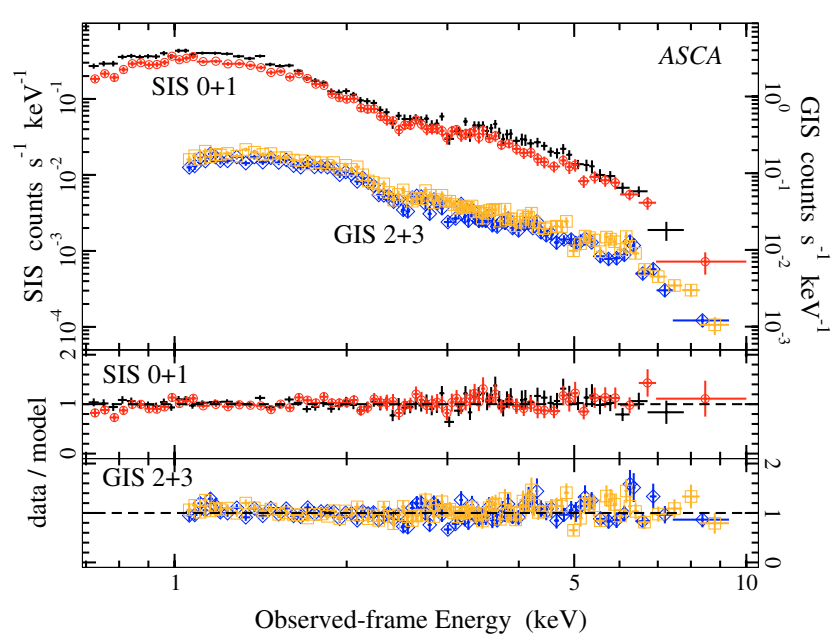

Fig. 3. Top panel: ASCA spectral data from the SIS0 (black crosses) and SIS1 (red open circles) instruments, and the two GIS instruments (blue diamonds and orange squares correspond to GIS2 and GIS3, respectively). The GIS flux density is given on the right axis. Middle panel: SIS residuals from the best-fit power-law plus Gaussian model (see text and Table 3 for details) fitted simultaneously with all ASCA instruments. Lower panel: same as middle panel, but for the GIS instruments. The data are binned for display purposes.

Table 3. Best-fit power-law plus Gaussian profile for the ASCA data. The ASCA instruments used in each fit are given in Col. (1), and the quality of the fit is shown in Col. (2). The power-law photon index is provided in Col. (3). The line parameters energy, line width, and equivalent width are given in Cols. (4)-(6), respectively.

\begin{tabular}{lccccc}
\hline \hline I) & $(2)$ & $(3)$ & $(4)$ & $(5)$ & $(6)$ \\
Instrument & $\chi_{v}^{2} /$ d.o.f. & $\Gamma$ & $\begin{array}{c}E \\
(\mathrm{keV})\end{array}$ & $\begin{array}{c}\sigma \\
(\mathrm{eV})\end{array}$ & $\begin{array}{c}E W \\
(\mathrm{eV})\end{array}$ \\
\hline SIS0+1 & $0.90 / 175$ & $2.02 \pm 0.03$ & $6.63_{-0.35}^{+0.17}$ & $<415$ & $305_{-32}^{+18}$ \\
GIS2+3 & $1.18 / 209$ & $2.10 \pm 0.03$ & $6.33_{-0.17}^{+0.12}$ & $<491$ & $420_{-42}^{+40}$ \\
SIS+GIS & $1.09 / 384$ & $2.06 \pm 0.02$ & $6.36_{-0.17}^{+0.14}$ & $<576$ & $384_{-20}^{+28}$ \\
\hline
\end{tabular}

evidence of intrinsic cold absorption from the ROSAT observations, the photoelectric absorption parameter was fixed to the value of the Galactic column.

Fitting the $2.5-10 \mathrm{keVspectra}$ with a power-law $(\Gamma \approx 1.96)$ resulted in a reasonable fit $\left(\chi_{\nu}^{2} /\right.$ d.o.f. $\left.=1.12 / 239\right)$; however positive residuals remained at $\sim 6.3 \mathrm{keV}$ in the observed-frame. Adding a Gaussian profile to the power-law model improved the fit considerably $\left(\Delta \chi^{2}=21.6\right.$ for 3 additional free parameters), suggesting emission from $\mathrm{Fe} \mathrm{K} \alpha$ at a rest frame energy of $E \approx 6.45 \mathrm{keV}$. Extrapolating the fit to $0.7 \mathrm{keV}$ in the SIS and $1 \mathrm{keV}$ in the GIS showed some residuals at lower energies. These residuals did not require additional model components to minimise, but could be adequately improved by simply allowing for a slight change in the slope of the current power-law. The resulting unabsorbed $2-10 \mathrm{keV}$ flux was $F \approx 1.1 \times 10^{-11} \mathrm{erg} \mathrm{cm}^{-2} \mathrm{~s}^{-1}$, and the corresponding intrinsic luminosity was $L \approx 2.1 \times 10^{43} \mathrm{erg} \mathrm{s}^{-1}$. The best-fit model is displayed in Fig. 3 and the parameters are given in Table 3. 


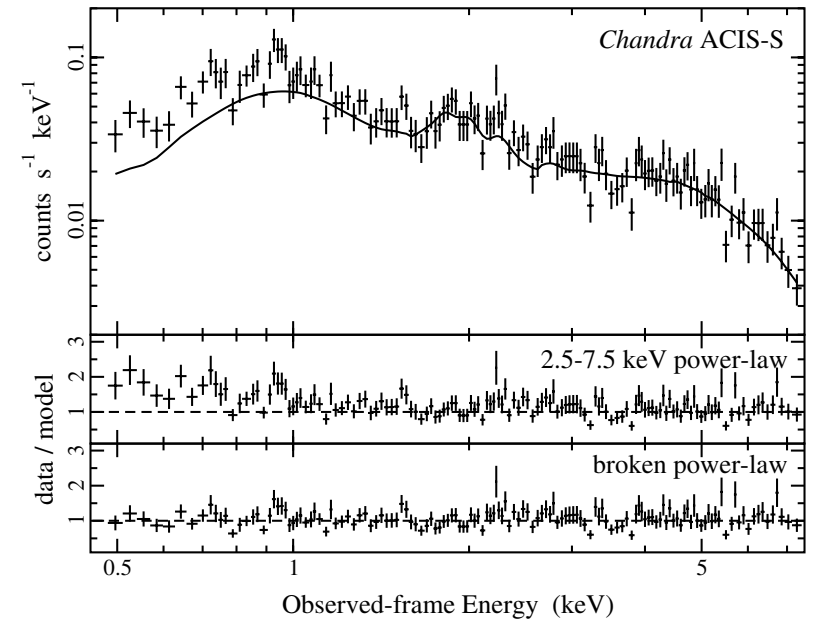

Fig. 4. The Chandra $0.5-7.5 \mathrm{keV}$ count spectra, fitted with a 2.5-7.5 keV power-law extrapolated to lower-energies, is shown in the top panel. The ratio from this fit is shown in the middle panel. Note the excess below $\sim 1 \mathrm{keV}$. In the bottom panel are the residuals (data/model) from fitting the spectra with a broken power-law (see text and Table 4 for details). The data are binned for display purposes.

\subsection{The Chandra spectra}

In order to minimise the effects of pile-up in the zeroth order spectrum, the pile-up model of Davis (2001) was used in all spectral fits. The parameters of this model component are give in Table 4.

As with the $A S C A$ analysis, we began by fitting the highenergy (2.5-7.2 keV) Chandra (0-order) data with a power-law $\left(\Gamma=2.10_{-0.34}^{+0.36}\right)$ modified by Galactic absorption. This resulted in a rather poor fit $\left(\chi_{v}^{2} /\right.$ d.o.f. $\left.=1.24 / 61\right)$, and there were no indication of possible emission features (Fig. 4).

Extrapolating the power-law down to $0.5 \mathrm{keV}$, showed evidence of a slight excess below $\sim 1 \mathrm{keV}$ (Fig. 4). Fitting the broadband continuum with just the single power-law (as was done to the $A S C A$ data) did not result in an entirely satisfactory fit (see Table 4 for broadband fit results).

The addition of a second continuum component, in the form of a blackbody or a broken power-law, improved the powerlaw model equally well $\left(\Delta \chi^{2}=8.3\right.$ for 2 additional free parameters). The unabsorbed $0.5-2 \mathrm{keV}$ and $2-10 \mathrm{keV}$ fluxes obtained from the broken power-law fit were $2.22 \times 10^{-11}$ and $2.31 \times 10^{-11} \mathrm{erg} \mathrm{cm}^{-2} \mathrm{~s}^{-1}$, respectively. The $2-10 \mathrm{keV}$ rest frame luminosity was $4.47 \times 10^{43} \mathrm{erg} \mathrm{s}^{-1}$.

The addition of a Gaussian profile did not significantly improve the fit $\left(\Delta \chi^{2}=1.5\right.$ for 3 additional parameters). Fixing a narrow $(\sigma=1 \mathrm{eV})$, Gaussian profile to $6.4 \mathrm{keV}$, as was found with $A S C A$, allowed estimation of a $90 \%$ upper-limit on the equivalent width of $E W<149 \mathrm{eV}$.

The best-fit models to the $0.5-7.5 \mathrm{keV}$ Chandra data are described in Table 4. The available statistics in the HEG and MEG are quite low. Nevertheless, application of the zeroth order best-fit model to the grating spectra resulted in comparable fits.
Table 4. Best-fit models to the Chandra data. In Col. (1) the adopted continuum model is given, and in Col. (2) the quality of fit. In Col. (3) the continuum model parameters, and in Col. (4) the parameters from the pile-up model are shown. The grade morphing $(\alpha)$ and the PSF fraction treated for pile-up ( $p s f$ ) are free parameters (see Davis 2001 for details).

\begin{tabular}{lccc}
\hline \hline $\begin{array}{l}\text { (1) } \\
\begin{array}{l}\text { Continuum } \\
\text { model }\end{array}\end{array}$ & $\begin{array}{c}(2) \\
\chi_{v}^{2} \text { d.o.f. }\end{array}$ & $\begin{array}{c}\text { Continuum } \\
\text { parameters }\end{array}$ & $\begin{array}{c}\text { Pile up } \\
\text { parameters }\end{array}$ \\
\hline power-law & $1.26 / 137$ & $\Gamma=2.24 \pm 0.21$ & $p s f=0.91_{-0.09}^{+0.04}$ \\
& & & $\sigma=0.43_{-0.10}^{+0.45}$ \\
\hline broken & $1.22 / 135$ & $\Gamma_{1}=2.52_{-0.30}^{+0.28}$ & $p s f=0.89_{-0.30}^{+0.05}$ \\
power-law & & $E_{b}=1.17_{-0.40}^{+0.74} \mathrm{keV}$ & $\sigma=0.32_{-0.12}^{+0.68}$ \\
& & $\Gamma_{2}=1.96 \pm 0.20$ & \\
\hline $\begin{array}{l}\text { blackbody } \\
\text { plus }\end{array}$ & $1.22 / 135$ & $k T=105 \pm 23 \mathrm{eV}$ & $p s f=0.89_{-0.23}^{+0.05}$ \\
power-law & & $\Gamma=1.96 \pm 0.23$ & $\sigma=0.34_{-0.12}^{+0.66}$ \\
\hline
\end{tabular}

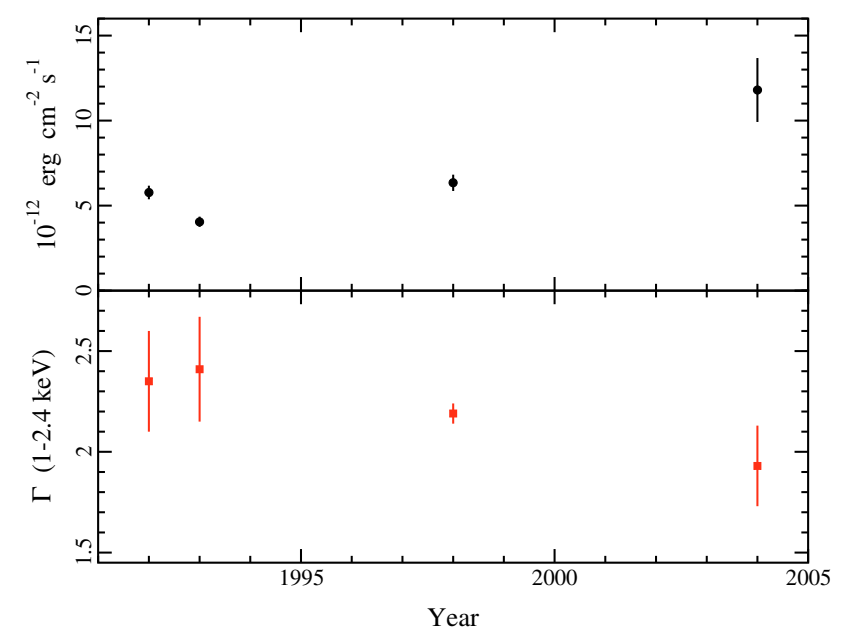

Fig. 5. Flux (upper panel) and spectral (lower panel) variability curves built from the four pointed observations utilised in this analysis. The energy range used to make the measurements is $1-2.4 \mathrm{keV}$ because it is the only band sampled by all the instruments. The photon index $(\Gamma)$ is obtained from fitting the $1-2.4 \mathrm{keV}$ range with a power-law, and the error bars in the flux are estimated from the uncertainties in the normalisation of the power-law at $1 \mathrm{keV}$.

\section{Timing properties}

\subsection{Long-term timing behaviour}

The four observations analysed here were spread over more than ten years; however the flux in the $1-2.4 \mathrm{keV}$ range, which is the only band sampled at every epoch, varied by less than a factor of three (see Fig. 5). The variability appears modest in comparison to other Seyferts (e.g. Markowitz \& Edelson 2004), but a fair comparison cannot be made given our poorly sampled long-term light curve.

Moreover, there is no significant spectral variability over this period. Within $90 \%$ uncertainties, the spectral slope between 1-2.4 keVis constant (Fig. 5). In addition, the spectral fits to the two ROSAT and Chandra data sets suggest that the low-energy $(E<1 \mathrm{keV})$ spectral form remains constant, and comparable to a power-law with a spectral index of $\Gamma \approx 2.5$. 


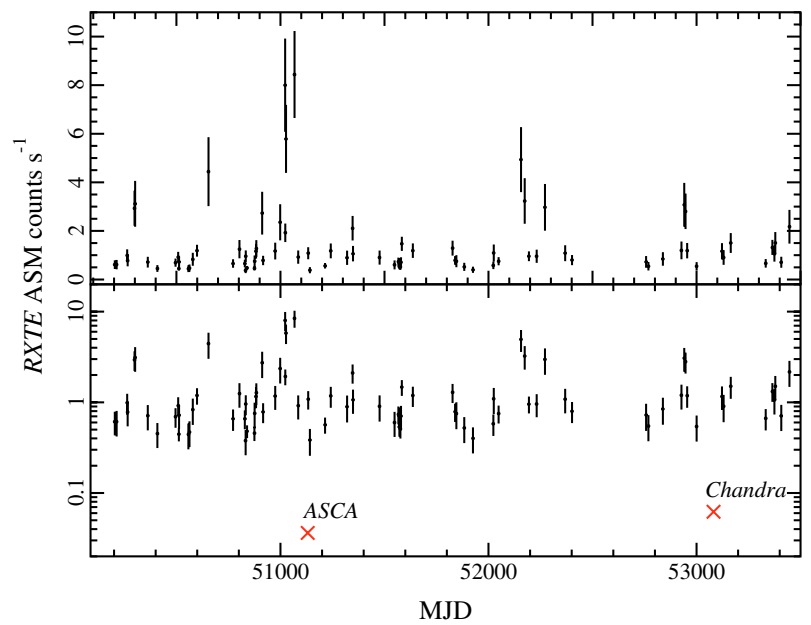

Fig. 6. Long-term RXTE ASM light curve constituting only data when Mrk 705 was detected above the $3 \sigma$ level. The light curve is shown in linear (top panel) and logarithmic (bottom panel) count $\mathrm{s}^{-1}$. The red $\mathrm{X}$ mark the estimated ASM count rate of Mrk 705 during the ASCA and Chandra observations.

Mrk 705 has been observed nearly daily with the $R X T E$ ASM since 1996 January. As of 2005 April 27 over 2600 observations were made. Mrk 705 has been detected above a $3 \sigma$ level about 85 times. In Fig. 6, the $>3 \sigma$ data points from the quick-look 2-10 keV light curve provided by the ASM/RXTE team are plotted. Using the ASCA and Chandra best-fit models, we have estimated an ASM count rate with WebPIMMS ${ }^{6}$, and over-plotted these estimates in Fig. 6. Since most of the time Mrk 705 is not significantly detected, it seems reasonable to assume that during the ASCA and Chandra observations (and likely the ROSAT observations as well), Mrk 705 was in a typical flux state. Assuming so, the ASM light curve demonstrates several examples of flaring events in which the $2-10 \mathrm{keV}$ flux increases by one, possibly even two orders of magnitude.

\subsection{Short-term timing behaviour}

The combined ASCA SIS0 and SIS1 light curves in a hard $(2-10 \mathrm{keV})$ and soft $(0.5-2 \mathrm{keV})$ band over the duration $(\sim 83 \mathrm{ks})$ of the observation are shown in Fig. 7 . On this time scale, both light curves fluctuate by $\pm 10 \%$ about the mean and are inconsistent with a constant according to a $\chi^{2}$-test $\left(\chi^{2}=99.0\right.$ and 40.0/12 d.o.f. for the soft and hard light curves, respectively). In addition, the hardness ratio between these two bands also shows variations over this period (lower panel of Fig. 7); however, there is no clear correlation between flux and spectral variability.

When examining the Chandra light curve, and the ASCA light curves during each telescope revolution, the fluctuations are greatly diminished (primarily due to statistics). For example, the $\sim 20 \mathrm{ks}$ Chandra light curve in $500 \mathrm{~s}$ bins was entirely consistent with a constant $\left(\chi_{v}^{2}<1\right)$. To compare the rapid variability in Mrk 705 to other AGN we calculated the excess variance $\left(\sigma_{\mathrm{rms}}^{2}\right)$ following Nandra et al. (1997), using $128 \mathrm{~s}$ binning of the $0.5-10 \mathrm{keVSIS}$ light curve. We estimate a value

\footnotetext{
${ }^{6}$ http://heasarc.gsfc.nasa.gov/Tools/w3pimms.html
}

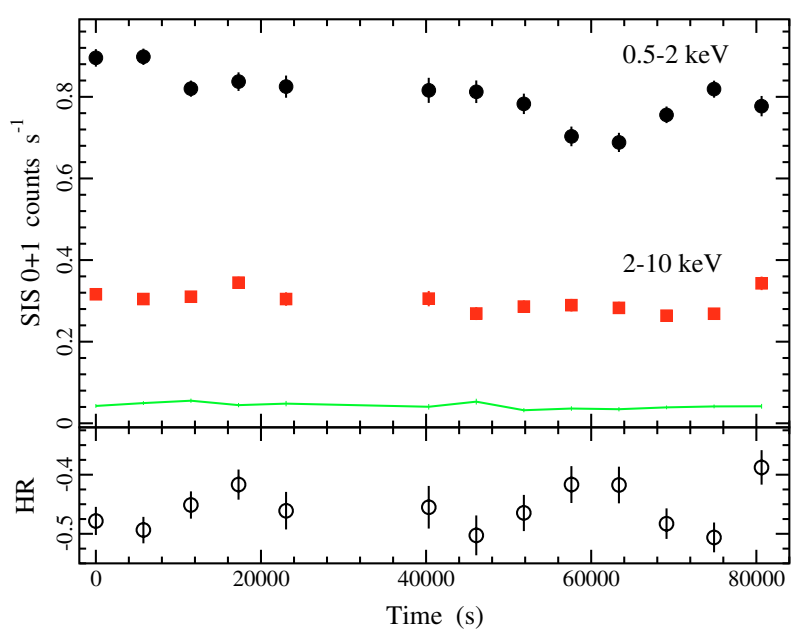

Fig. 7. Top panel: ASCA soft (0.5-2 keV) and hard (2-10 keV) combined SIS light curves in $5760 \mathrm{~s}$ bins. The background light curve in the $0.5-10 \mathrm{keVband}$ is marked by the green solid line. Lower panel: The hardness ratio ( $H R$, see footnote 1$)$, is plotted against elapsed time. In this case, $H$ and $S$ are as defined in the top panel.

of $\sigma_{\text {rms }}^{2} \approx 0.26 \times 10^{-2}$. In comparing the excess variance derived for Mrk 705 with Fig. 3 of Leighly (1999), in which both NLS1 and BLS1 are plotted, it is clear that the weak variations of Mrk 705 on short time scales are typical of BLS1 of comparable luminosities.

\section{Conclusions}

We present spectra from four pointed observations of the borderline NLS1, Mrk 705, utilising data from ROSAT, ASCA and Chandra which span over ten years. In addition, we also present the 2-10 keVlight curve obtained with the RXTE ASM. The main results of this analysis are the following:

- The X-ray position is well correlated with the optical and radio positions of Mrk 705 . There is an unidentified radio source located within $30^{\prime \prime}$ of Mrk 705; however, this object is not detected with Chandra, suggesting that the X-ray emission measured with ROSAT and ASCA is dominated by Mrk 705.

- Comparing the measured flux during the pointed observations with the long-term ASM light curve, the four spectra of Mrk 705 appear to have been obtained during flux states typical for the source. The flux during these pointed observations is comparable within a factor of $\sim 3$.

- In the ROSAT and Chandra observations there is indication of an additional low-energy emission component, which appears constant in spectral form $(\Gamma \approx 2.5)$ at all three epochs.

- The high-energy (2-10 keV) photon index does not vary significantly between the ASCA and Chandra observations, although the flux increases by about a factor of two. An Fe $\mathrm{K} \alpha$ emission with $E W \approx 380 \mathrm{eV}$ is detected in the $A S C A$ spectrum. A line is not statistically required in the Chandra data, and the upper-limit on the equivalent width of a narrow $6.4 \mathrm{keV}$ line is $E W<149 \mathrm{eV}$. The measured equivalent widths at the two epochs are in agreement with 
the flux of the line remaining constant while the continuum flux varies. This suggests that the line probably originates in distant material such as the torus.

- The long-term light curve obtained with the RXTE ASM indicates that large amplitude flaring-type events are rare in Mrk 705, but they do occur.

- During the $\sim 83 \mathrm{ks} A S C A$ observation, Mrk 705 does show fluctuations of about $\pm 10 \%$ in the hard, soft and hardness ratio variability curves. On shorter time scales the variability is diminished.

The optical properties of Mrk 705 place it on the borderline distinguishing NLS1 and BLS1. The X-ray properties examined at various epochs over more than ten years do not allow a clearer definition, but suggest that Mrk 705 is more similar to BLS1. The rather flat slope of the soft-excess, weak short-term variability and neutral iron line (Porquet et al. 2004) are consistent with BLS1 X-ray properties. However, there is some evidence from the RXTE light curve of, albeit rare, large amplitude fluctuations, which have been seen frequently in some NLS1 (e.g. Boller et al. 1997; Brandt et al. 1999).

Deeper studies of samples and individual objects which seem to exhibit properties of both NLS1 and BLS1 could be fruitful in establishing a more accurate defining scheme, as well as revealing the underlying physics.

Acknowledgements. This research has made use of the Tartarus (Version 3.0) database, created by Paul O'Neill and Kirpal Nandra at Imperial College London, and Jane Turner at NASA/GSFC. Tartarus is supported by funding from PPARC, and NASA grants NAG5-7385 and NAG5-7067. This work has also made use of results provided by the ASM/RXTE teams at MIT and at the RXTE SOF and GOF at NASA's GSFC. Many thanks are due to the referee for a quick report.

\section{References}

Arnaud, K. 1996, in Astronomical Data Analysis Software and Systems, ed. G. Jacoby, \& J. Barnes, ASP Conf. Ser., 101, 17

Baskin, A., \& Laor, A. 2004, MNRAS, 350, 31

Boller, Th., Brandt, W. N., \& Fink, H. 1996, A\&A, 305, 53
Boller, Th., Brandt, W. N., Fabian, A. C., \& Fink, H. 1997, MNRAS, 289, 393

Boroson, T. A., \& Green, R. F. 1992, ApJS, 80, 109

Botte, V., Ciroi, S., Rafanelli, P., \& Di Mille, F. 2004, AJ, 127, 3168

Brandt, W. N., Boller, Th., Fabian, A. C., \& Ruszkowski, M., 1999, MNRAS, 303, 58

Clements, E. D. 1981, MNRAS, 197, 829

Davis, J. 2001, ApJ, 562, 575

Dewangan, G., \& Griffiths, R. 2005, ApJ, 625, 31

Elvis, M., Lockman, F. J., \& Wilkes, B. J. 1989, AJ, 97, 777

Ferrari, A. 1998, ARA\&A, 36, 539

Goodrich, R. W. 1989, ApJ, 342, 234

Grupe, D. 1996, Ph.D. Thesis, Univ. Göttingen

Grupe, D. 2004, AJ, 127, 1799

Grupe, D., \& Mathur, S. 2004, ApJ, 606, 41

Leighly, K. 1999, ApJS, 125, 297

Markowitz, A., \& Edelson, R. 2004, ApJ, 617, 939

Mathur, S. 2000, MNRAS, 314, 17

Mathur, S., Kuraszkiewicz, J., \& Czerny, B. 2001, NewA, 6, 321

Mathur, S., Grupe, D. 2005a, A\&A, 432, 463

Mathur, S., \& Grupe, D. 2005b, ApJ, accepted [arXiv: astro-ph/0507624]

Nandra, K., George, I. M., Mushotzky, R. F., Turner, T. J., \& Yaqoob, T. 1997, ApJ, 476, 70

Osterbrock, D. E., \& Pogge, R. W. 1985, ApJ, 297, 166

Porquet, D., Reeves, J., O'Brien, P., \& Brinkmann, W. 2004, A\&A, 422,85

Pfeffermann, E., Briel, V. G., Hippmann, H., et al. 1987, Proc. SPIE, 733,519

Puchnarewicz, E. M., Mason, K. O., Cordova, F. A., et al. 1992, MNRAS, 256, 589

Reeves, J., Wynn G., O'Brien, P., \& Pounds, K. 2002, MNRAS, 336, 56

Véron-Cetty, M.-P., Véron, P., \& Gonçalves, A. 2001, A\&A, 372, 730

Voges, W., Aschenbach, B., Boller, Th., et al. 1999, A\&A, 349, 389

Wang, T., Brinkmann, W., \& Bergeron, J. 1996, A\&A, 309, 81

White, R., Becker, R., Helfand, D., \& Gregg, M. 1997, ApJ, 475, 479

Williams, R., Pogge, R., \& Mathur, S. 2002, AJ, 124, 3042

Williams, R., Mathur, S., \& Pogge, R., 2004, ApJ, 610, 737

Worrall, D., \& Birkinshaw, M. 2004, [arXiv: astro-ph/0410297]

Zimmermann, H., et al. 1994, MPE Rep., 257 